\title{
Literature Review and Case-Series of Atypical Anatomic Locations for Parathyroid Glands
}

\author{
Stefan Vidovich, $B S^{1}$, Johanna Wickemeyer, $M D^{1}$, Pouyan Kheirkhah, $M D^{2}$ and Ari Rubenfeld, \\ $M D^{1 *}$
}

${ }^{1}$ Department of Otolaryngology, University of Illinois at Chicago, Chicago, IL, USA

${ }^{2}$ Department of Pathology, University of Illinois at Chicago, Chicago, IL, USA

\begin{abstract}
Specialty area: Endocrine surgery.

Introduction: Essential to successful parathyroid surgery is understanding of the possible anatomic variations in parathyroid gland location. In this case-series, we highlight three patients with uniquely located parathyroid glands as noted during primary neck exploration.

Methods: This is a case-series that details three different patients' health histories, diagnostic workups, intraoperative findings, and surgical pathology analyses. All three patients were evaluated and treated with parathyroidectomy for hyperparathyroidism between May and September of 2020. All data was collected from patient electronic medical records at UIHealth - University of Illinois at Chicago and de-identified in December of 2020.

Results: The first case demonstrated an inferiorly descended left superior parathyroid gland in the mediastinum. The second case exhibited bilateral intrathymic inferior parathyroid. The third case demonstrated a parathyroid adenoma intimately associated with the left vagus nerve, deep in the carotid sheath.
\end{abstract}

Conclusion: These three unique cases demonstrate the variations in parathyroid anatomy that may be encountered prior to and during parathyroid surgery. Increased vigilance for parathyroid ectopy amongst endocrine surgeons in the preoperative imaging and intraoperative settings affords an opportunity to maximize success of primary neck exploration.

Keywords

Endocrine surgery, Parathyroid glands, Ectopy

\section{Introduction}

Primary hyperparathyroidism (PHPT) is the most common type of hyperparathyroidism (HPT) with an estimated prevalence of $0.86 \%$ in the United States [1]. It is predominantly caused by solitary benign parathyroid adenomas, which represent roughly $80 \%$ of cases [2]. Further causes of PHPT include multiglandular disease as a result of four-gland parathyroid hyperplasia and multiple benign parathyroid adenomas. Parathyroid carcinoma accounts for fewer than $1 \%$ of PHPT cases [3]. Secondary HPT can be due to various disease states including chronic kidney disease (CKD), vitamin D deficiency and malabsorptive disorders, among others [4]. Prevalence of secondary HPT among CKD and vitamin $D$ deficient patients is particularly high $[5,6]$. Tertiary HPT is almost exclusively a complication in severe CKD or post-renal transplant patients [7].

HPT in high-income countries such as the US, Canada and those of Western Europe is predominantly asymptomatic, due to routine serologic screening recognizing electrolyte disturbances before they become problematic [8]. Roughly $20-30 \%$ of patients are symptomatic, with the most common manifestation being recurrent nephrolithiasis [4]. Skeletal manifestations include bone pain, osteitis fibrosa cystica and pathologic bone fractures [8]. Also seen are proximal muscle weakness, fatigue, abdominal pain, nausea, pancreatitis and psychiatric changes such as increased anxiety [4,8]. Symptoms of secondary and tertiary HPT are similar to those of PHPT [7].

The superior and inferior parathyroid glands are derived

*Corresponding author: Ari Rubenfeld, MD, Department of Otolaryngology, University of Illinois at Chicago, Chicago, IL, USA

Accepted: September 08, 2021

Published online: September 10, 2021

Citation: Vidovich S, Wickemeyer J, Kheirkhah P, et al. (2021) Literature Review and Case-Series of Atypical Anatomic Locations for Parathyroid Glands. J Surgical Endocrinol 3(2):101-106 
Citation: Vidovich S, Wickemeyer J, Kheirkhah P, et al. (2021) Literature Review and Case-Series of Atypical Anatomic Locations for Parathyroid Glands. J Surgical Endocrinol 3(2):101-106

from the third and fourth pharyngeal pouches, respectively, and begin their migratory descent around the sixth week of embryogenesis. The superior parathyroid glands have a shorter distance, relative to the inferior glands, to their final position. In contrast the inferior parathyroid glands travel along with the thymic primordium, arriving at their typical anatomic position behind the inferior aspect of the thyroid gland [9]. It is possible for ectopic parathyroid glands to be found anywhere along both migratory paths with an estimate of $6-22 \%$ of parathyroid glands found ectopically [10-13]. Inferior parathyroid glands are more susceptible to deviating from a normal anatomical location due to the significantly longer migration path they follow $[9,11]$. Ectopic superior parathyroid glands most often rest in the tracheoesophageal groove or retroesophageal space. Meanwhile, ectopic inferior glands are frequently localized within the thymus or thyroid gland [11-14]. Ectopic parathyroid glands have been noted to rest along the hypoglossal nerve [15], vagus nerve [1618], or within the submandibular region [19]. In this study, we discuss three cases of ectopic parathyroid tissue found at initial parathyroid exploration.

\section{Case Presentations}

\section{Intrathymic parathyroid glands}

Patient 1 is a 47-year-old male whose medical history includes end-stage renal disease on hemodialysis, hypertension, and osteoporosis complicated by multiple pathologic bone fractures. He was referred to Otolaryngology clinic by Nephrology due to chronic fatigue, generalized body pain and weakness, joint stiffness, and anxiety. Physical examination demonstrated no abnormal findings, with no evidence of palpable neck masses. He was then sent to Endocrinology, and a diagnosis of tertiary hyperparathyroidism was established as seen in Table 1 under Patient 1. Upon return to Otolaryngology clinic, he was formally evaluated and scheduled for surgery. Radiographic imaging included DEXA scan which demonstrated possible grade 1 fracture at L4 and T-score of -2.8 (Table 2). The patient was scheduled for bilateral neck exploration (BNE) without localizing imaging.

Intraoperatively, there was a firm, highly calcified lesion identified in the right inferior quadrant where the inferior

Table 1: Preoperative and immediate postoperative PTH values for patients 1 and 2 .

\begin{tabular}{|l|l|l|}
\hline Serum Test (normal range/unit) & Patient 1 & Patient 2 \\
\hline Pre-op PTH (12-88 pg/mL) & $\mathbf{3 3 3 5}(\mathbf{H})$ & $\mathbf{9 8 2}(\mathbf{H})$ \\
\hline Immediate post-op PTH & 11 & 3 \\
\hline
\end{tabular}

Table 2: DEXA scan bone density results.

Bone Density: Exam date 08/26/2019

\begin{tabular}{|l|l|l|l|}
\hline Region & BMD $\left(\mathbf{g} / \mathbf{c m}^{2}\right)$ & T-score & Z-score \\
\hline AP Spine (L1-L4) & 0.778 & -2.8 & -2.6 \\
\hline
\end{tabular}

World Health Organization criteria for BMD impression classify patients as Normal ( $\mathrm{T}$-score at or above -1.0), Osteoperia ( $\mathrm{T}$-score between -1.0 and -2.5), or Osteoporosis (T-score at or below -2.5). parathyroid gland would be expected in its usual position, superficial to the recurrent laryngeal nerve (RLN). Final pathology confirmed this as hyperplastic parathyroid tissue. Immediately deep to the right $\mathrm{RLN}$ in a region consistent with usual location for superior parathyroid gland, was another significantly calcified lesion. Final pathology confirmed hyperplastic tissue. On the left side, the inferior thyroid pole was elevated and revealed an enlarged, highly calcified, multilobulated lesion later confirmed as hyperplastic parathyroid tissue, and felt to be consistent in its location with an inferior parathyroid gland. The dissection continued superiorly where the left RLN was identified. There was no evidence of the left parathyroid gland in its usual location. Dissection continued superiorly to the carotid body and then retropharyngeally, but no gland was appreciated. Inferiorly, a calcified lesion was localized within thymic tissue and confirmed to be hyperplastic parathyroid tissue. Given that the left inferior parathyroid gland was prior identified in its usual location, and parathyroid tissue was not identified in other ectopic locations superiorly, this was felt to be the left superior parathyroid gland. Immediate postoperative PTH level was $11 \mathrm{pg} / \mathrm{mL}$, down from $3335 \mathrm{pg} / \mathrm{mL}$.

Patient 2 is a 28-year-old female with a history of endstage renal disease following failed kidney transplant. She had been on hemodialysis for 13 years posttransplant and suffered from mixed renal osteodystrophy complicated by pathologic left femur fracture, secondary hyperparathyroidism, heart failure with reduced ejection fraction (20-25\%) and severe hypertension. She initially presented to the emergency department with severe left leg pain secondary to pathologic left femur fracture. On physical examination in the Head and Neck clinic, there were no notable neck masses. Laboratory results are elaborated in Table 1 under Patient 2. Radiographic imaging included Tc99 Sestamibi parathyroid scan (Sestamibi), thyroid ultrasound (UST), whole-body radionuclide bone scan (NM bone scan) and numerous $\mathrm{X}$-rays and CT scans. Single-photon emission computed tomography (SPECT) was unable to be performed owing to Patient 2 having claustrophobia. Sestamibi (Figure 1) revealed no definitive scintigraphic findings. UST performed by Radiology demonstrated multiple subcentimeter thyroid nodules but was unable to visualize parathyroid glands. NM bone scan confirmed left proximal femur fracture along with heterogenous appearance of multiple pelvic bones. Past $\mathrm{x}$-rays and CT scans all demonstrated chronic bone changes consistent with underlying renal osteodystrophy. She was sent for surgical evaluation by Endocrinology.

This patient underwent a bilateral neck exploration. After incision and identification of anatomical landmarks, initial exploration of the left inferior thyroid pole revealed no evidence of parathyroid tissue. Further dissection toward the left cricothyroid joint exposed an enlarged lesion confirmed as parathyroid tissue by frozen pathology, presumably the left superior parathyroid gland. Moving to the right neck, an enlarged right superior lesion was identified beneath the right superior thyroid pole. It was confirmed on frozen pathology as parathyroid tissue. Tracing the right RLN inferiorly, dissection continued throughout the central neck 
Citation: Vidovich S, Wickemeyer J, Kheirkhah P, et al. (2021) Literature Review and Case-Series of Atypical Anatomic Locations for Parathyroid Glands. J Surgical Endocrinol 3(2):101-106

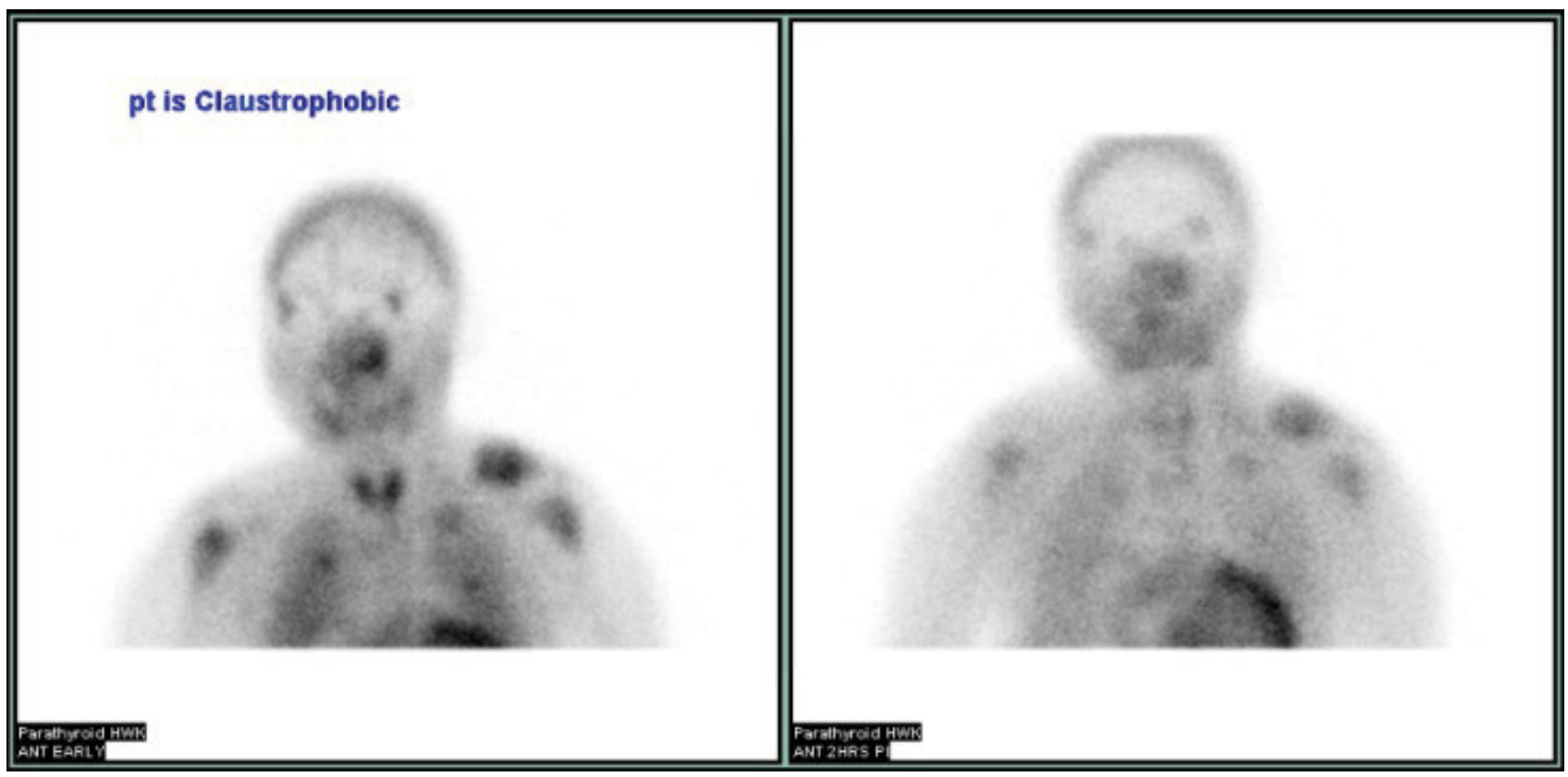

Figure 1: Sestamibi scan with increased uptake in region of the thyroid, but otherwise no specific findings.

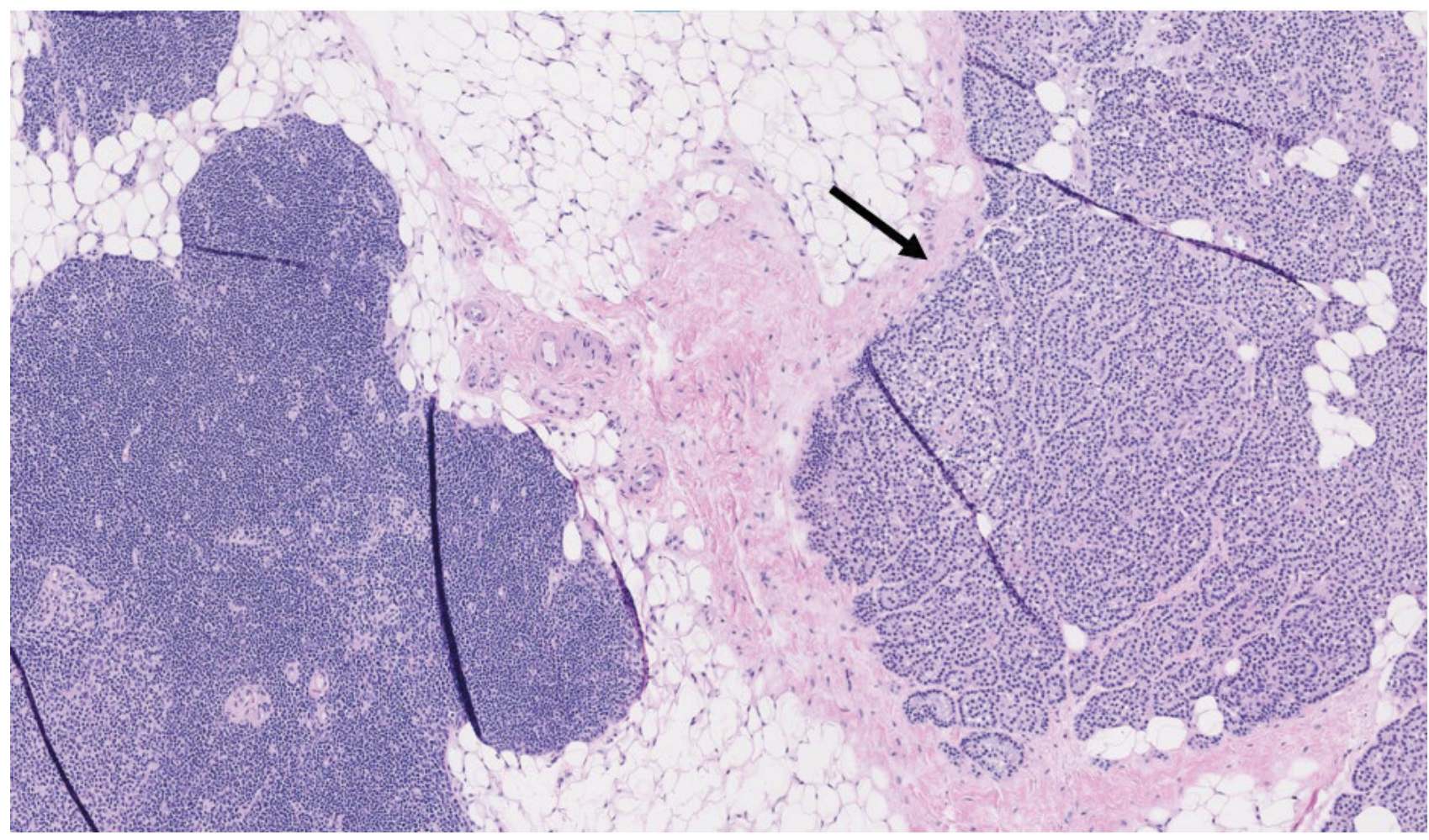

Figure 2: H\&E-stained slide demonstrating chief cells organized in a pseudofollicular pattern, interspersed with adipose cells, and surrounded by a thin fibrous capsule (arrow), consistent with parathyroid tissue. Opposite is a dense lobulated pocket of T-lymphocytes surrounded by a thin layer of epithelial cells, consistent with thymic cortical tissue. High-power (16.5x) magnification.

toward the innominate artery. It was below the clavicles that thymic tissue was removed superior to the innominate artery, exposing two adenomatous lesions suspicious for parathyroid tissue. Frozen pathology corroborated that both lesions were of parathyroid origin and were within the thymus gland (Figure 2 and Figure 3). Immediate postoperative PTH testing was $3 \mathrm{pg} / \mathrm{mL}$ with values normalizing to $57 \mathrm{pg} / \mathrm{mL}$, decreased from a preoperative level of $982 \mathrm{pg} / \mathrm{mL}$.

\section{Paravagal parathyroid gland}

Patient 3 is a 43-year-old male with history of vitamin D deficiency and follicular B-cell lymphoma treated with 


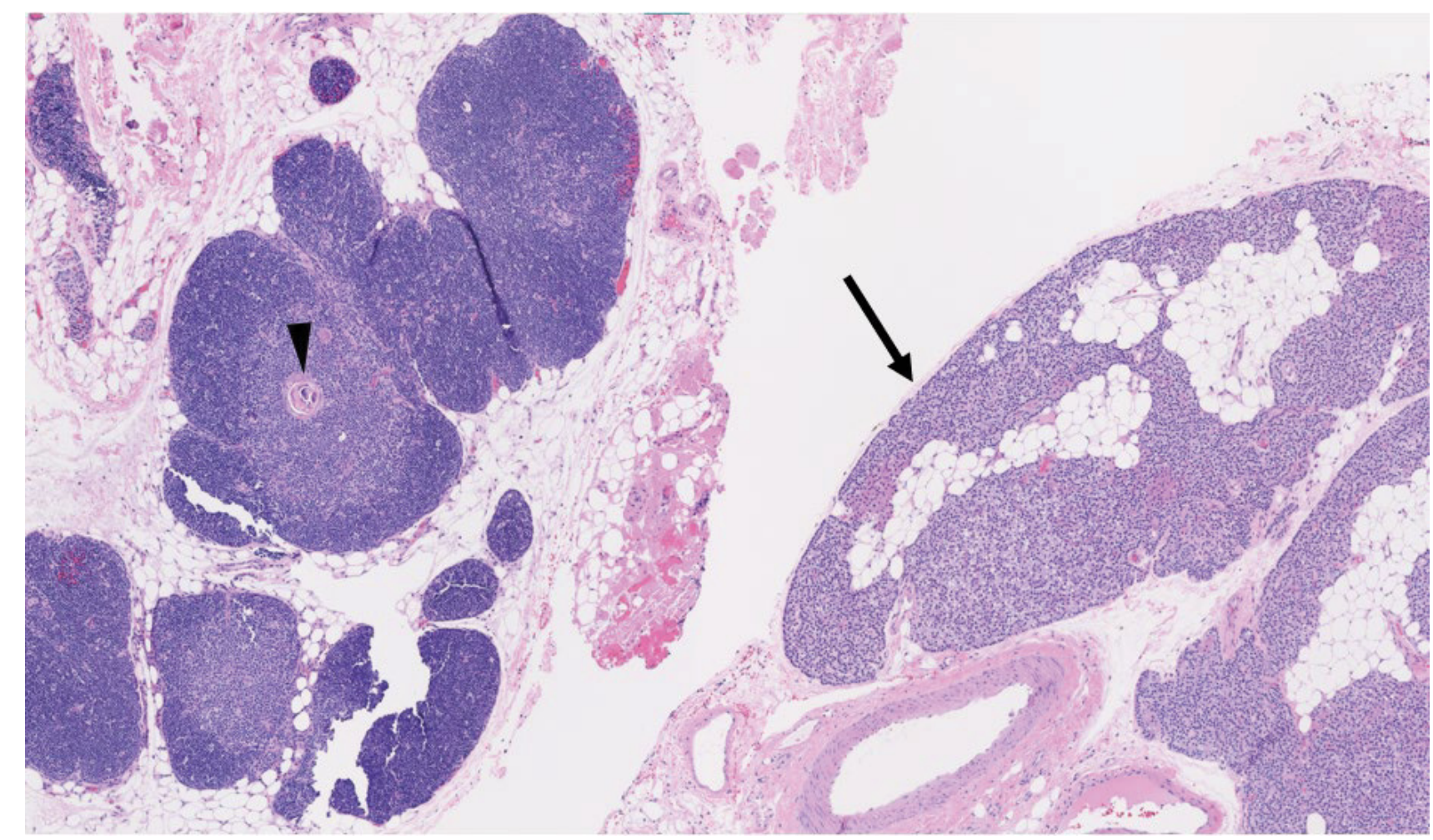

Figure 3: H\&E-stained slide demonstrating parathyroid tissue (arrow) opposite thymic tissue, as evidenced by Hassell's corpuscle (triangle) within thymic medullary tissue. High-power (10.9x) magnification.

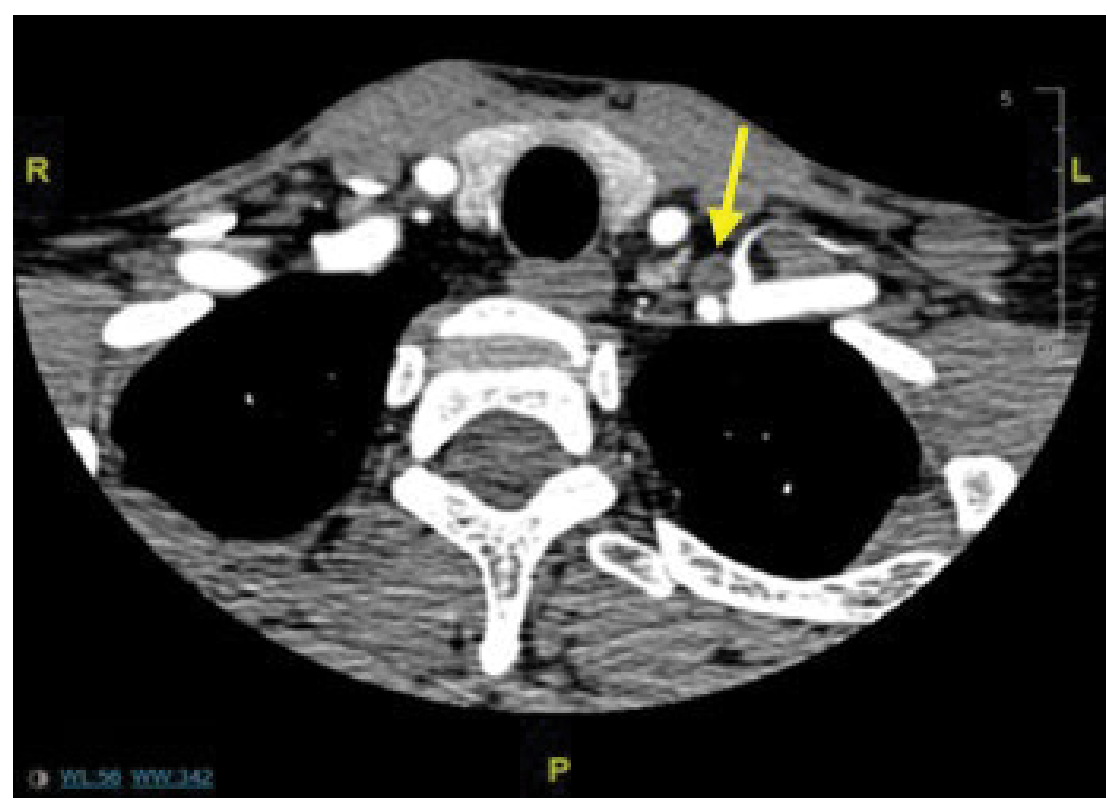

Figure 4: CT neck with evidence of soft tissue nodule (arrow) between the left common carotid and subclavian arteries.

chemotherapy. Hypercalcemia was an incidental finding during workup for lymphoma. He had kidney stones several years prior. Apart from fatigue as a result of chemotherapy, he denied any constitutional, gastric or musculoskeletal symptoms prior to starting therapy. Workup conferred a diagnosis of PHPT. Laboratory results were reviewed and showed elevated calcium at $11.6 \mathrm{mg} / \mathrm{dL}$ and inappropriately high PTH of 93 pg/mL. Radiographic studies ordered included a DEXA scan, helical CT neck with and without contrast, and NM parathyroid scan with SPECT. DEXA scan (Table 3) revealed osteoporosis. CT neck (Figure 4) showed a $1.3 \times$ $1.9 \times 3.3 \mathrm{~cm}$ soft tissue nodule between the left common carotid and left subclavian arteries. This was suspicious for parathyroid adenoma, but given the surprising location, a physiologic study was appropriate. NM parathyroid with SPECT demonstrated no definitive findings with minimal 


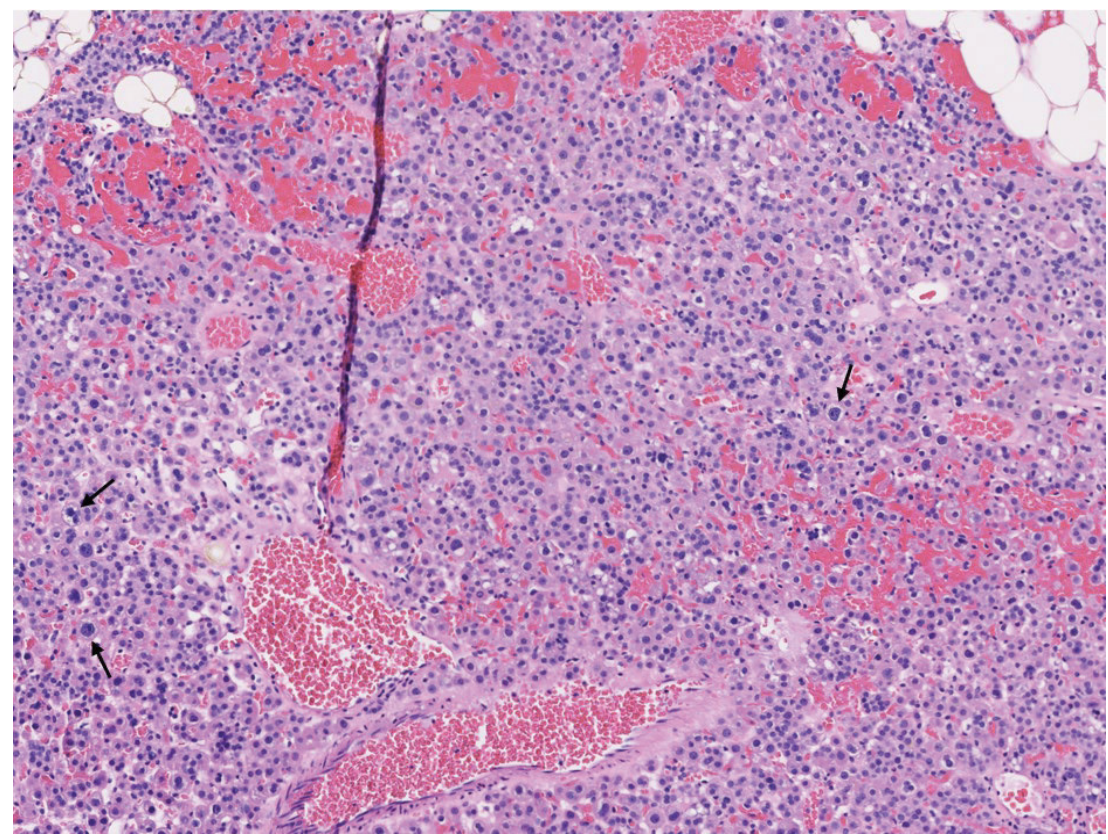

Figure 5: H\&E-stained slide demonstrating left inferior PTH adenoma with evidence of nuclear atypia (arrows). High-power (21.4x) magnification.

Table 3: DEXA scan results revealing significant osteoporosis.

\begin{tabular}{|l|l|l|l|l|}
\hline Region & BMD $\mathbf{( g / \mathbf { c m } ^ { 2 } )}$ & T-score & Z-score & \\
\hline AP Spine (L1-L4) & 0.792 & -2.7 & -3.5 & TBS 1.349 \\
\hline Femeral Neck (Left) & 0.507 & -3.1 & -2.7 & \\
\hline Total Hip (Left) & 0.637 & -2.6 & -2.5 & \\
\hline
\end{tabular}

World Health Organization criteria for BMD impression classify patients as Normal (T-score at or above -1.0), Osteoperia (T-score between -1.0 and -2.5 ), or Osteoporosis (T-score at or below -2.5).

TBS: Trabecular bone score

radiotracer activity on delayed images leading to a broadened differential including atypical parathyroid adenoma versus treated B-cell lymphoma versus ectopic thyroid tissue.

Having localized a lesion via multiple imaging modalities, parathyroid exploration was merited, with the intention of first exploring the paravagal region. PTH immediately prior to incision was measured at $158 \mathrm{pg} / \mathrm{mL}$. The left common carotid artery (CCA) was identified and dissected along its medial edge. Upon gentle medial retraction of the left CCA, the solitary lesion was revealed to be deep to and wrapped over by the vagus nerve. The mass was adherent to the floor of the posterior neck. The vagus nerve was carefully traced and isolated from the enveloping lesion. Nerve stimulation (laryngeal monitoring) post-extirpation confirmed that left vagus function was intact. Intraoperative PTH monitoring at five, 10, and 20 minutes resulted in an appropriate decrease in PTH of 29,26 , and $22 \mathrm{pg} / \mathrm{mL}$, respectively. Surgical pathology confirmed the left lower neck lesion to be an atypical PTH adenoma (Figure 5).

\section{Discussion}

Ectopic parathyroid glands can present in a multitude of locations. Current literature has estimated that anywhere from $17.0 \%$ to $62 \%$ of ectopic parathyroid glands will be found within the thymus or thyrothymic ligament [12,13,20,21]. The first two cases presented above exhibit intrathymic parathyroid gland ectopy. Based upon literature review here, each case has specific qualities that have yet to be reported.

The first case was unique as it was presumably the left superior parathyroid gland, rather than inferior gland as would be expected, that presented within the thymus. This certainly challenges accepted embryologic expectations. The inferior parathyroid glands and thymus share a common embryological origin at the third pharyngeal pouch [10], which makes it more likely to suspect an inferior gland had localized to the thymus. As an alternate theory, both the left superior and inferior parathyroid glands could have shifted downward simultaneously. This is not, however, supported by our intraoperative findings of the left inferior PTH gland within its usual position deep to the inferior thyroid artery and ventral to the recurrent laryngeal nerve. To the best of our knowledge, this is the first documented case of a superior parathyroid gland localizing to the thymus.

Regarding the second patient, we were surprised to find that bilateral inferior parathyroid glands were intrathymic. While not an uncommon ectopic site, we believe this is the first reported case of bilateral inferior, non-supernumerary parathyroid glands to be found within the thymus.

For our final patient, the left-sided parathyroid adenoma was found to be closely associated with the left vagus nerve. Due to its close approximation, we initially believed the ectopic gland to be intravagal. Intravagal parathyroid glands are quite rare with only 12 cases reported in the literature, though Lack, et al. [22] estimated an incidence of $6 \%$ based on vagus nerve dissection in 32 infants postmortem. Final 
Citation: Vidovich S, Wickemeyer J, Kheirkhah P, et al. (2021) Literature Review and Case-Series of Atypical Anatomic Locations for Parathyroid Glands. J Surgical Endocrinol 3(2):101-106

pathologic analysis revealed no evidence of neural tissue within the adenoma. Furthermore, it would have been less likely for an intravagal adenoma to be found in the lower neck as multiple studies and case reports have demonstrated that most intravagal parathyroid glands are found at or above the level of the carotid bifurcation $[17,18,23]$.

These three cases reinforce the impressive diversity surrounding parathyroid gland anatomy and should highlight the importance of staying vigilant for possible ectopy. Further differentiating these cases is the fact that all ectopic glands were identified during primary exploration, contrasting from the fact that many cases of ectopy are identified in patients requiring multiple neck explorations for persistent hyperparathyroidism $[20,21]$.

We believe surgeons should anticipate ectopy both in the interpretation of preoperative imaging and intraoperatively. From a diagnostic standpoint, continued use of Sestamibi and US thyroid is prudent, particularly if there is an interest in the physiologic avidity of the lesion. Intraoperatively, surgeons should be aware of common ectopic sites for both superior and inferior parathyroid glands. If a gland cannot be identified in usual anatomic locations, a sequential explorative approach should be employed to search for a possible ectopic gland, as is the historical expectation of parathyroid surgery. Being cognizant of extreme anatomical variations is advantageous as it may decrease morbidity associated with persistent hyperparathyroidism as well as potential complications associated with secondary neck surgery.

\section{Conclusion}

In conclusion, this case series further enriched existing literature regarding the variable nature of parathyroid gland anatomy, and it brings to light some highly unusual anatomic circumstances. We encourage surgeons to continue utilizing appropriate preoperative parathyroid imaging as well as maintaining an intimate knowledge of parathyroid gland ectopy to improve surgical management of parathyroid disease.

\section{Acknowledgements}

We would like to thank the UIC Pathology Department for contributing through the provision of patient parathyroid tissue samples.

\section{Ethical Approval}

An exemption was furnished by the UIC College of Medicine Institutional Review Board.

\section{Conflicts of Interest}

The authors have no conflicts of interest or financial relations to disclose.

\section{References}

1. Press DM, Siperstein AE, Berber E, et al. (2013) The prevalence of undiagnosed and unrecognized primary hyperparathyroidism: A population-based analysis from the electronic medical record. Surgery 154: 1232-1238.
2. Baloch ZW, Livolsi VA (2015) Parathyroids: Morphology and pathology. In: Bilezikian JP, Marcus R, Levine MA, Marcocci C, Silverberg SJ, The parathyroid. ( $3^{\text {rd }}$ edn), Elsevier, USA, 23-36.

3. Cetani F, Marcocci C (2015) Parathyroid carcinoma. In: Bilezikian JP, Marcus R, Levine MA, Marcocci C, Silverberg SJ, The parathyroids, ( $3^{\text {rd }}$ edn), Elsevier, USA, 409-422.

4. Fraser WD (2009) Hyperparathyroidism. Lancet 374: 145-158.

5. Bureo JC, Arévalo JC, Antón J, et al. (2015) Prevalence of secondary hyperparathyroidism in patients with stage 3 and 4 chronic kidney disease seen in internal medicine. Endocrinol Nutr 62: 300-305.

6. Mosekilde L (2005) Vitamin D and the elderly. Clin Endocrinol (Oxf) 62: 265-281.

7. Jamal SA, Miller PD (2013) Secondary and tertiary hyperparathyroidism. J Clin Densitom 16: 64-68.

8. BilezikianJP, Banderia L, KhanA, etal.(2018)Hyperparathyroidism. Lancet 391: 168-178.

9. Bae J, Skinner MA (2010) Endocrine disorder and tumors. In: Ashcraft KW, Holcomb GW, Murphy JP, Ostlie DJ, Ashcraft's Pediatric Surgery 5th edition. Elsevier, USA, 1037-1054.

10. Wang C (1976) The anatomic basis of parathyroid surgery. Ann Surg 183: 271-275.

11. Phitayakorn R, McHenry CR (2006) Incidence and location of ectopic abnormal parathyroid glands. Am J Surg 191: 418-423.

12. Madhuchhanda $R$, Mazeh $H$, Chen $H$, et al. (2013) Incidence and localization of ectopic parathyroid adenomas in previously unexplored patients. World J Surg 37: 102-106.

13. Eslamy HK, Ziessman HA (2008) Parathyroid scintigraphy in patients with primary hyperparathyroidism: $99 \mathrm{mTc}$ sestamibi SPECT and SPECT/CT. Radiographics 28: 1461-1476.

14. Noussios G, Anagnostis P, Natsis K (2012) Ectopic parathyroid glands and their anatomical, clinical and surgical implications. Exp Clin Endocrinol Diabetes 120: 604-610.

15. Karvounaris DC, Symeonidis N, Triantafyllou A, et al. (2010) Ectopic parathyroid adenoma located inside the hypoglossal nerve. Head Neck 32: 1273-1276.

16. Daruwalla J, Sachithanandan N, Andrews D, et al. (2015) Ectopic intravagal parathyroid adenoma. Head Neck 37: E200-E204.

17. Chan TJ, Libutti SK, McCart JA, et al. (2003) Persistent primary hyperparathyroidism caused by adenomas identified in pharyngeal or adjacent structures. World J Surg 27: 675-679.

18. Pawlik TM, Richards M, Giordano TJ, et al. (2001) Identification and management of intravagal parathyroid adenoma. World J Surg 25: 419-423.

19. Kong Y, Ge SY, Shang W, et al. (2019) Ectopic parathyroid adenoma in the submandibular region: A case report. Br J Oral Maxillofac Surg 57: 1150-1152.

20. Schneider R, Waldmann J, Ramaswamy A, et al. (2011) Frequency of ectopic and supernumerary intrathymic parathyroid glands in patients with renal hyperparathyroidism: Analysis of 461 patients undergoing initial parathyroidectomy with bilateral cervical thymectomy. World J Surg 35: 1260-1265.

21. Taterra D, Wong L, Vikse J, et al. (2019) The prevalence and anatomy of parathyroid glands: A meta-analysis with implications for parathyroid surgery. Langenbecks Arch Surg 404: 63-70.

22. Lack EE, Delay S, Linnoila I (1988) Ectopic parathyroid tissue within the vagus nerve. Arch Pathol Lab Med 112: 304-306.

23. Takimoto $T$, Okabe $Y$, Ito $M$, et al. (1989) Intravagal parathyroid adenoma. J Laryngol Otol 103: 704-706. 